\title{
Kinetic Monte Carlo for Process Simulation: First Principles Calibrated Parameters for $\mathrm{BO}_{2}$
}

\author{
Pierre-Louis Julliard \\ STMicroelectronics \\ Crolles, France \\ pierre-louis.julliard@st.com \\ Frederic Monsieur \\ STMicroelectronics \\ Crolles, France \\ frederic.monsieur@st.com \\ Layla Martin-Samos \\ CNR-IOM \\ Trieste, Italy \\ marsamos@iom.cnr.it
}

\author{
Antoine Jay \\ LAAS-CNRS \\ Toulouse, France \\ ajay@laas.fr
}

\author{
Nicolas Guitard \\ STMicroelectronics \\ Crolles, France \\ nicolas.guitard@st.com
}

\author{
Denis Rideau \\ STMicroelectronics \\ Crolles, France \\ denis.rideau@st.com
}

\author{
Miha Gunde \\ $L A A S-C N R S$ \\ Toulouse, France \\ miha.gunde@laas.fr \\ Thomas Cabout \\ STMicroelectronics \\ Crolles, France \\ thomas.cabout@st.com
Fuccio Cristiano
$L A A S-C N R S$
Toulouse, France
cfuccio@laas.fr

\author{
Nicolas Salles \\ CNR-IOM \\ Trieste, Italy \\ salles@iom.cnr.it \\ Sylvain Joblot \\ STMicroelectronics \\ Crolles, France \\ sylvain.joblot@st.com \\ Anne Hemeryck \\ $L A A S$-CNRS \\ Toulouse, France \\ anne.hemeryck@laas.fr
}

\begin{abstract}
The formation energies and diffusion paths of $\mathbf{O}_{i}$, $\mathrm{O}_{2 i}$ and $\mathrm{BO}_{2}$ complex in silicon are determined using $a b$ initio calculations and used to calibrate a Kinetic Monte Carlo. The aim is to simulate the formation of the $\mathrm{BO}_{2}$ complex in Technology Computer Aided Design tools.

Index Terms-Boron-Oxygen complex, Kinetic Monte Carlo, process simulation, TCAD
\end{abstract}

\section{INTRODUCTION}

Formation of Boron-Oxygen complex $\left(\mathrm{BO}_{2}\right)$ is known to be a major issue in $\mathrm{Cz}$-silicon photovoltaic cells: the $\mathrm{BO}_{2}$ induces a drastic reduction of the minority lifetime carrier [1], which is detrimental for the photoconversion efficiency. It is also detrimental in image sensors since $\mathrm{BO}_{2}$ is reported to introduce a deep energy level into the bandgap [2] that can act as an active recombination center giving rise to a significant increase of the dark current. As reported in [3] optimizing the process can avoid the formation of $\mathrm{BO}_{2}$ complex.

The purpose of this study is first to identify the fundamental mechanisms underlying the formation of $\mathrm{BO}_{2}$ complex in silicon material by means of ab initio calculations. And later, to exploit the results to accurately calibrate the Sentaurus Process KMC solver [4]. It will be shown that $\mathrm{BO}_{2}$ complex formation requires a detailed description of the diffusion of isolated oxygen $\mathrm{O}_{i}$, the formation and possible dissociation of $\mathrm{O}_{2 i}$, and the association and dissociation of $\mathrm{O}_{2 i}$ with the Boron doping element as detailed in Section III-A. In Section III-B, with the new first principle-based parameters, the solver predicts diffusion coefficients in better agreement with available experimental data.

978-1-6654-0685-7/21/\$31.00@2021 European Union

\section{Simulation AND CAlibRation METhod}

Each KMC event is governed by an Arrhenius' law $D \times$ $\exp \left(E_{a c} / k_{B} T\right)$ that requires the knowledge of its entropic prefactor $D$ and of its activation barrier $E_{a c}$.

The events needed to form the $\mathrm{BO}_{2}$ complex, are the diffusion of isolated $\mathrm{O}_{i}$ atoms, their agglomeration in molecular $\mathrm{O}_{2 i}$ and the formation of $\mathrm{BO}_{2}$. Hence, four species have to be implemented in the KMC code: $\mathrm{O}_{i}, \mathrm{O}_{2 i}, \mathrm{~B}_{s} O_{2 i}$ and $\mathrm{B}_{i} \mathrm{O}_{2 i}$, where $\mathrm{B}_{s} O_{2 i}$ and $\mathrm{B}_{i} \mathrm{O}_{2 i}$ respectively refer to a $\mathrm{BO}_{2}$ complex in which the boron atom is in a substitutional [5] and interstitial site [6].

These species have been relaxed in a 216-atoms cubic supercell of silicon atoms with periodic boundary conditions in different charge states until forces on each atom are less than $10^{-4}$ Ry/a.u. Based on the formation energy of such species, we identify the one most likely to exist in the material and which will be likely to migrate [6]. Total energy calculations have been performed within Density Functional Theory (DFT) [7], [8] using the Perdew-Burke-Ernzerhof functional (PBE [9]) as implemented in Quantum Espresso [10]. The HeydScuseria-Ernzerhof (HSE [11]) functional has been used for the evaluation of the formation energy at each charge state. PAW and Vanderbilt pseudopotentials were used for the PBE and for the HSE calculations, respectively. The plane-wave basis cut-off was set to $50 \mathrm{Ry}$. The sample of the Brillouin zone was performed at the $\Gamma$ point only with PBE and with a Monkhorst-Pack [12] grid of $2^{3}$ k-points for the HSE. Activation barriers $E_{a c}$ have been determined using ARTn refine-saddle mode [13]. $D$ have been fitted to match the diffusion coefficients measured in [14] [15]. Binding energy $E_{b}$ for a given system is determined with the difference 
between the formation energy $E_{f}$ of the system and the formation energies of the elements composing the system isolated in a Si-supercell: $E_{b}\left(O_{2 i}\right)=E_{f}\left(O_{2 i}\right)-2 \times E_{f}\left(O_{i}\right)$.

\section{RESULTS}

\section{A. DFT Investigation}

$\mathbf{O}_{i}$ specie: formation and migration - We found that the most stable structure for $\mathrm{O}_{i}$ is the $C_{1 h}$, in agreement with [16] $\left(\mathrm{O}_{i}\right.$-A in Fig. 1.A). The charge state is found to be neutral independently of the Fermi level value as shown in Fig. 1. The calculated migration energy for $\mathrm{O}_{i}$ is $2.25 \mathrm{eV}$. The saddle point of the migration is shown in Fig. 1.B. Experimentally, the high temperature diffusion of $\mathrm{O}_{i}$ species in silicon is associated to a migration barrier of $2.53 \mathrm{eV}$ [17].

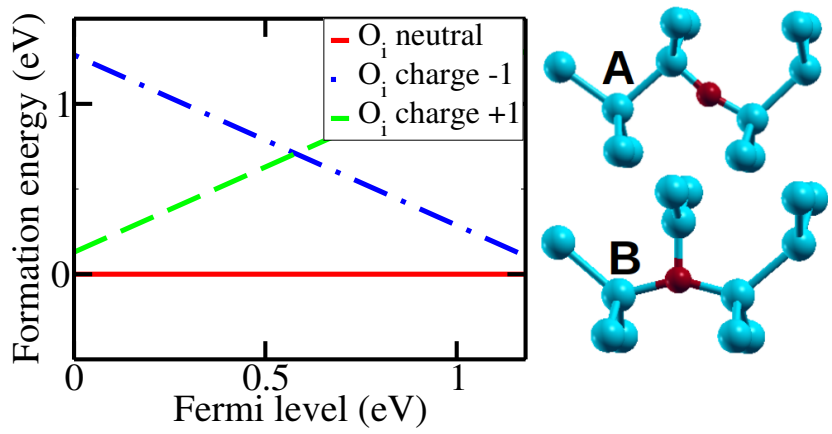

Fig. 1. Left: $\mathrm{O}_{i}$ formation energy for different charge states. Right: Atomic configuration for $C_{1 h}\left(\mathrm{O}_{i}-\mathrm{A}\right)$ and saddle point of migration path $\left(\mathrm{O}_{i}-\mathrm{B}\right)$. Red and blue spheres are respectively $\mathrm{O}$ and $\mathrm{Si}$ atoms.

$\mathbf{O}_{2 i}$ specie: formation, dissociation and migration Staggered $\mathrm{O}_{2 i}$-st and $\mathrm{O}_{2 i}^{++}$squared configurations are the most discussed structures discussed in the literature [18]. In our calculations, the staggered $\mathrm{O}_{2 i}$-st configuration (Fig. 2 topright) exhibits the lowest energy (Fig. 2 top-left) in comparison to the formation energy of the $\mathrm{O}_{2 i}^{++}$squared structure $\left(\mathrm{O}_{2 i}{ }^{-}\right.$ sq in Fig.2). Therefore the hypothesis of a migration by the means of $\mathrm{O}_{2 i}^{++}$squared structure found in [18] is unlikely to occur even at low Fermi level energy. The migration energy is thus calculated for a neutral charge state. The barrier energy is calculated to be $1.40 \mathrm{eV} . \mathrm{O}_{2 i}$-sq is revealed to be the saddle point along the migration path. Binding energy of $\mathrm{O}_{2 i}(-0.30$ $\mathrm{eV}$ ) calculated with PBE is consistent with experimental values (Tab. I) and indicates that the formation of molecular $\mathrm{O}_{2 i}$ specie is a favorable process.

TABLE I

COMPARISON OF THE CALCULATED ACTIVATION BARRIERS $E_{a c}$ AND BINDING ENERGY $E_{b}$ (IN EV) AND THE EXPERIMENTAL ONES. THE PREFACTOR $D\left(\mathrm{~s}^{-1}\right)$ IS ADJUSTED EMPIRICALLY TO FIT EXPERIMENTAL DATA FROM [14] [15].

\begin{tabular}{|l|c|c|c|c|c|}
\hline \multirow{2}{*}{} & \multicolumn{3}{|c|}{ DFT-PBE } & \multicolumn{2}{c|}{ Exp. } \\
\cline { 2 - 6 } & $\boldsymbol{E}_{a c}$ & $\boldsymbol{E}_{b}$ & $\boldsymbol{D}$ & $\boldsymbol{E}_{a c}$ & $\boldsymbol{E}_{b}$ \\
\hline $\mathrm{O}_{i}$ & 2.25 & - & $3.10^{14}$ & $2.53[17]$ & - \\
\hline $\mathrm{O}_{2 i}$ & 1.40 & -0.30 & $4.10^{15}$ & $1.5[15]$ & $-0.30[19]$ \\
\hline
\end{tabular}
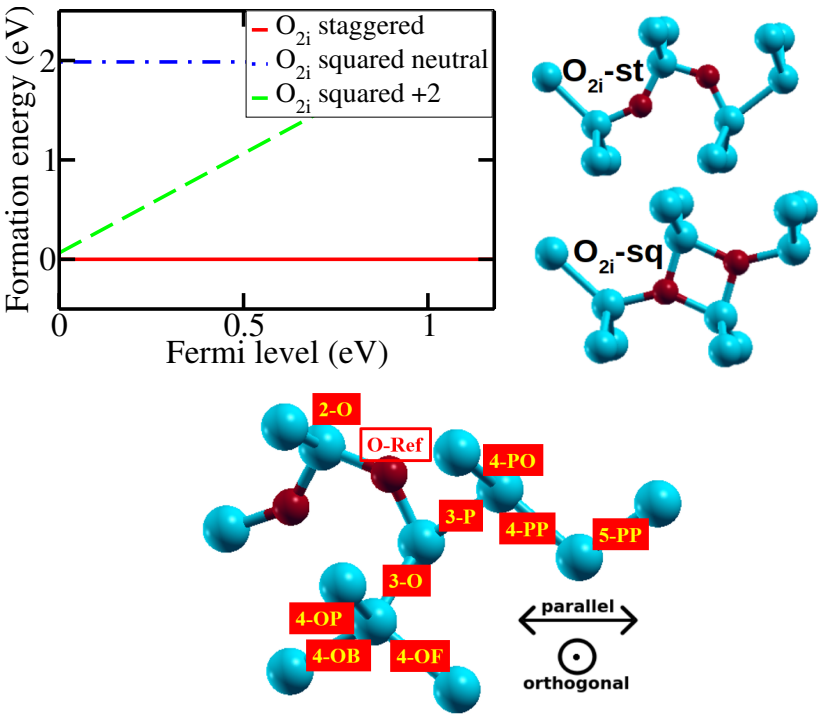

Fig. 2. Top left: $\mathrm{O}_{2 i}$ formation energy for squared $\left(\mathrm{O}_{2 i}\right.$-sq) and staggered $\left(\mathrm{O}_{2 i}\right.$-st) structures in different charge states. Top right: $\mathrm{O}_{2 i}$-st atomic configuration. $\mathrm{O}_{2 i}$-sq is the saddle point of migration path of $\mathrm{O}_{2 i}$-st. Bottom: Mapping of the local environment involved during the dissociation of the $\mathrm{O}_{2 i}$ st toward the formation of two isolated $\mathrm{O}_{i}$. Red boxes represent the different interstitial sites where $\mathrm{O}_{i}$ from $\mathrm{O}$-Ref can migrate. The number and the letter in the boxes indicate the distance between Si neighbors and the direction of the migration (P Parallel, O Orthogonal) respectively. Corresponding activation barriers are given in Tab. II.

An exhaustive characterization of the activation barriers of the dissociation/association mechanisms of $\mathrm{O}_{2 i}$-st is necessary to correctly identify, then predict and simulate at what temperature $\mathrm{O}_{2 i}$-st is formed and how often it dissolves. To understand the interaction between the oxygen atoms and how it affects the activation barriers, one $\mathrm{O}_{i}$ atom is taken away from the atomic configuration $\mathrm{O}_{2 i}$-st to the next interstitial sites as depicted in Fig. 2 bottom panel. All the energy barriers associated to the dissociation mechanisms of the $\mathrm{O}_{2 i}$-st as a function of the nearest neighbors influence are given in Tab. II. Note that the calculated activation barriers associated to the energy gains provided in Tab. II allow a description of the formation of $\mathrm{O}_{2 i}$-st from two isolated $\mathrm{O}_{i}$ according to back reactions. Such an approach is carried out until the total energy of the system is equal to that of two isolated oxygen atoms, meaning that the two atoms are no longer in interaction, i.e. until the fourth Si neighbors.

To dissociate $\mathrm{O}_{2 i}$-st, we observe that the activation barriers of diffusion occurring in the direction parallel to the two $\mathrm{O}$ atoms of structure $\mathrm{O}_{2 i}$-st are always smaller than in the other direction: $\mathrm{O}_{i}$ initially on O-Ref will successively migrate toward sites 3-P, 4-PP... The reorientation of the structure $\mathrm{O}_{2 i}$ st on another plane $\left(\mathrm{O}_{i}\right.$ on site O-Ref moving to site 2-O on Fig. 2) requires a larger activation barrier of $2.45 \mathrm{eV}$. We reveal that the diffusion of $\mathrm{O}_{2 i}$-st is highly anisotropic. In the fourth neighbor position, the $\mathrm{O}_{i}$ atoms are still influencing each other. In this zone where the two $\mathrm{O}_{i}$ atoms interact, the two $\mathrm{O}_{i}$ atoms will have to tendency to cluster to form the 
TABLE II

ACTIVATION BARRIERS ( $\mathrm{E}_{a c}$ ) FOR THE DISSOCIATION OF $\mathrm{O}_{2 i}$-ST REFERRING TO CONFIGURATIONS LABELLED IN FIG. 2 BOTTOM PANEL. $\Delta E$ IS THE ENERGY DIFFERENCE BETWEEN THE INITIAL AND FINAL CONFIGURATIONS. ENERGIES ARE IN EV.

\begin{tabular}{|c|c|c|}
\hline \multicolumn{3}{|c|}{ From O-Ref to } \\
\hline site & $E_{a c}$ & $\Delta E$ \\
\hline $2-\mathrm{O}$ & 2.45 & 0.00 \\
\hline $3-\mathrm{P}$ & 1.82 & 0.60 \\
\hline $3-\mathrm{O}$ & 2.12 & 0.14 \\
\hline
\end{tabular}

\begin{tabular}{|c|c|c|}
\hline \multicolumn{3}{|c|}{ From site 3-O to } \\
\hline site & $E_{a c}$ & $\Delta E$ \\
\hline 4-OP & 2.24 & 0.17 \\
\hline $4-\mathrm{OF}$ & 2.36 & -0.10 \\
\hline $4-\mathrm{OB}$ & 2.28 & 0.06 \\
\hline
\end{tabular}

\begin{tabular}{|c|c|c|}
\hline \multicolumn{3}{|c|}{ From site 3-P to } \\
\hline site & $E_{a c}$ & $\Delta E$ \\
\hline $4-\mathrm{PP}$ & 1.38 & -0.12 \\
\hline $4-\mathrm{PO}$ & 1.67 & 0.48 \\
\hline
\end{tabular}

$\mathrm{O}_{2 i}$-st, rather than to separate. Beyond this interaction limit, the behavior of oxygen can be considered as an isolated $\mathrm{O}_{i}$ atom.

For an accurate predictive modeling of $\mathrm{O}_{2 i}$-st dissociation, we demonstrate the importance of considering the existing interaction between oxygen atoms even at long distances. We also identify the interaction limit of these oxygen atoms. Furthermore, this DFT-based study reveals that the dissociation (and formation) is highly anisotropic, which could be critical in the thermally activated regime.

$\mathrm{BO}_{2}$ complex: stability and formation - Structural relaxations confirm that the more stable configurations for $\mathrm{BO}_{2}$ complex are the $\mathrm{B}_{s} \mathrm{O}_{2 i}$ and $\mathrm{B}_{i} \mathrm{O}_{2 i}$ defects as obtained in [5] and [6]. Formation energies for the $\mathrm{B}_{s} \mathrm{O}_{2 i}$ defect demonstrate that the $\mathrm{B}_{s} \mathrm{O}_{2 i}$ in $\mathrm{B}$ configuration is dominant for a Fermi level below $0.22 \mathrm{eV}$ whereas the A configuration is more stable above (Fig. 3).

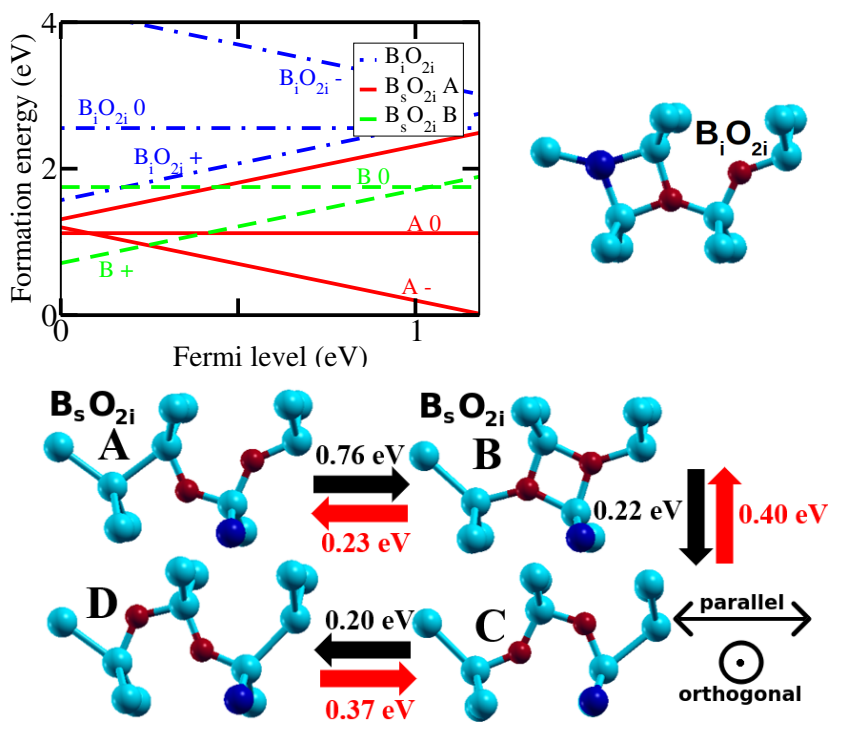

Fig. 3. Top left: formation energies for $B_{s} \mathrm{O}_{2 i}$ and $\mathrm{B}_{i} \mathrm{O}_{2 i}$ in their more favorable charge state. Top right : atomic configuration of $\mathrm{B}_{i} \mathrm{O}_{2 i}$. Bottom: Minimum Energy Path of the migration of $\mathrm{O}_{2 i}$-st from a $\mathrm{B}_{s} \mathrm{O}_{2 i}$ complex (A). Boron atoms are represented by dark blue spheres.
In the following, we focus on the $\mathrm{B}_{s} \mathrm{O}_{2 i}$ complex formation. This structure is likely to have a high migration barrier because of the stability of the boron atom in a substitutional position. Boron diffusion is only shown when associated with the presence of a vacancy [20]. Then, comparing the total energy between the $\mathrm{B}_{s} \mathrm{O}_{2 i}$ in configuration $\mathrm{A}$ and isolated $\mathrm{O}_{2 i}$-st and $\mathrm{B}_{s}^{-}$configuration indicates that the formation of $\mathrm{B}_{s} \mathrm{O}_{2 i}$ is a favorable process $(-1.09 \mathrm{eV})$.

We consider that the dissociation of the $\mathrm{B}_{s} \mathrm{O}_{2 i}$ complex occurs through the departure of $\mathrm{O}_{2 i}$ from the $\mathrm{B}_{s} \mathrm{O}_{2 i}$ complex. As in the case of $\mathrm{O}_{2 i}$, a detailed study of the dissociation of $\mathrm{B}_{s} \mathrm{O}_{2 i}$ should be investigated as a function of the interaction distance between $\mathrm{O}_{2 i}$ and $\mathrm{B}$ atom. Because of the large number of possibilities for dissociation, based on the findings of $\mathrm{O}_{2 i}$ diffusion described previously, we initially assume that $\mathrm{O}_{2 i}$ diffusion occurs in the plane containing the two oxygen atoms (in the $\mathrm{P}$ direction), consistent with other study [5]. The minimum energy path of the corresponding migration is shown in Fig. 3-Bottom part. The activation barrier to transform the $\mathrm{B}_{s} \mathrm{O}_{2 i}$-A configuration into the $\mathrm{B}_{s} \mathrm{O}_{2 i}$ - $\mathrm{B}$ configuration (when $\mathrm{O}_{2 i}$ is no longer symmetric about the $\mathrm{B}$ atom) is large by 0.76 $\mathrm{eV}$. Once the complex is in the $\mathrm{B}_{s} \mathrm{O}_{2 i}-\mathrm{B}$ configuration, the dissociation of $\mathrm{B}_{s} \mathrm{O}_{2 i}$ - $\mathrm{B}$ is slightly in favor of the formation of $\mathrm{B}_{s} \mathrm{O}_{2 i}-\mathrm{C}\left(E_{a c}\right.$ are $0.22 \mathrm{eV}$ from $\mathrm{B}$ to $\mathrm{C}$ vs. $0.23 \mathrm{eV}$ from $\mathrm{B}$ to $\mathrm{A})$. The same trend is observed in the next step between the $\mathrm{B}_{s} \mathrm{O}_{2 i}-\mathrm{C}$ and $\mathrm{B}_{s} \mathrm{O}_{2 i}$-D configuration $\left(E_{a c}\right.$ are $0.20 \mathrm{eV}$ from $\mathrm{C}$ to $\mathrm{D} v s$. $0.40 \mathrm{eV}$ from $\mathrm{C}$ to $\mathrm{B}$ ). Thus these calculations are consistent with the formation of the $\mathrm{B}_{s} \mathrm{O}_{2 i}-\mathrm{A}$ configuration, which once formed is very stable. The formation of this complex is nevertheless dependent on the area around the Boron atom which tends to make this complex not form immediately. This formation time linked to the tendency of $\mathrm{O}_{2 i}$ not to be systematically attracted by the boron atom, must be considered to give a predictive account of the formation of $\mathrm{B}_{s} \mathrm{O}_{2 i}$.

\section{B. KMC process simulation}

$\mathbf{O}_{i}$ and $\mathbf{O}_{2 i}$ calibration - For this purpose we calculated the oxygen diffusivity with the view of comparing KMC prediction to measurement of [14] and [15]. Oxygen diffusivity is simulated with KMC by following the diffusion of a buried oxygen-rich silicon layer in a bulk silicon during annealing at different temperatures. Oxygen diffusion in silicon is assumed to be driven by $\mathrm{O}_{i}$ migration for temperature above $700^{\circ} \mathrm{C}$. Between $400^{\circ} \mathrm{C}$ and $700^{\circ} \mathrm{C}$, the experimental diffusivity shows an enhancement. Several hypotheses exist to explain the diffusion acceleration: a fast diffusion of $\mathrm{O}_{2 i}$ complex is one of the most cited explanations [17].

For an efficient use, KMC codes use a single energy barrier to simulate a dissociation event. Dissociation energy for $\mathrm{O}_{2 i}$ is determined as done in [21]: $E_{\text {dissociation }}\left(O_{2 i}\right)=E_{b}\left(O_{2 i}\right)+$ $E_{m}\left(O_{i}\right)$. The DFT values used for the KMC calibration are given in Tab. I. As can be seen in Fig. 4, the DFT calibratedKMC exhibits a clear enhanced diffusion between $400^{\circ} \mathrm{C}$ and $700^{\circ} \mathrm{C}$. We notice a discrepancy between the experimental data and the KMC simulations results, especially in the region 
of the enhanced diffusion. This observation can have several explanations. The $\mathrm{O}_{2 i}$ driven diffusion is dependent on the migration energy of the $\mathrm{O}_{2 i}$-st but also on its interaction not favoring the dissociation of $\mathrm{O}_{2 i}$. As we detailed in previous section, the multiple pathways shown in Fig. 2 should be considered to better represent the diffusion of $\mathrm{O}_{2 i}$. A single dissociation and migration event is certainly not sufficient to accurately describe the increased diffusion.

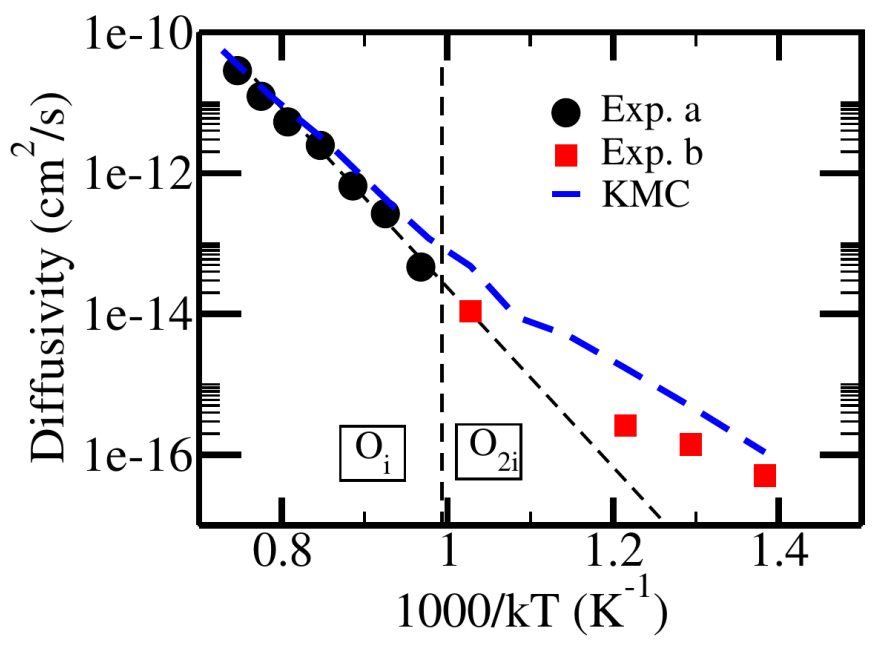

Fig. 4. KMC prediction for oxygen diffusivity obtained with the events of Table I. Comparison with the experimental diffusion coefficients from [14] (a) and [15] (b). Dashed vertical line shows the separation between the $\mathrm{O}_{i}$ driven diffusion regime and the $\mathrm{O}_{2 i}$ diffusion regime and dashed inclined line is guideline for the eyes for the $\mathrm{O}_{i}$ mediated diffusion.

Implementation of $\mathbf{B}_{s} \mathbf{O}_{2 i}$ complex - It can be noted incidentally that the boron parameters have been calibrated elsewhere [21]. To implement the $\mathrm{BO}_{2}$ complex formation, following method could be applied: the emission of an $\mathrm{O}_{2 i}$ from a $\mathrm{B}_{s} \mathrm{O}_{2 i}$ defect can be estimated using the same method as the emission of an $\mathrm{O}_{i}$ from an $\mathrm{O}_{2 i}$, by knowing the energies of the $\mathrm{B}$ atoms and $\mathrm{O}_{2 i}$ in a $\mathrm{Si}$ supercell. In such way, the dissociation mechanism to form $\mathrm{a} \mathrm{B}_{s}^{-}$and an $\mathrm{O}_{2 i}$ from a negatively charged $\mathrm{A}$ configuration of $\mathrm{B}_{s} \mathrm{O}_{2 i}$ must overcome a binding energy of $1.09 \mathrm{eV}$ and the migration energy of the $\mathrm{O}_{2 i}$ given in Tab I. These two barriers lead to an activation energy of $2.49 \mathrm{eV}$ for the dissociation mechanism.

As we have already proven before, this calibration method has again several limitations. A KMC code that uses only one activation barrier per event cannot adequately handle the effect of the interactions at more or less long distance that we observe for the dissociation (or formation) of the $\mathrm{B}_{s} \mathrm{O}_{2 i}$ complex.

\section{CONCLUSION}

DFT calculations provide accurate values of activation energies for atomistic events as needed for TCAD tool calibration. The KMC calibrated with $a b$ initio inputs reproduces enhanced diffusion of oxygen by the mean of $\mathrm{O}_{2 i}$ kinetics. We also show that by a conventional way, it is also possible to implement the process simulation of the $\mathrm{BO}_{2}$ formation using the DFT calculated parameters for oxygen species and $\mathrm{BO}_{2}$.
In the idea of calibrating the KMC sentaurus code as built today, we have proposed in this paper the possibility of implementing a single activation barrier as required by the code that we have calculated in DFT. This approach has the advantage of being fast and easy to use. However, we showed through a detailed study at the ab initio scale, that this approach has many drawbacks.

To solve this problem, an off-lattice KMC seems to be a promising tool. We are currently developing such an off lattice KMC [22] in order to use the complete library of possible events we have identified in this paper. The goal of the off lattice KMC simulations will be to determine the correct prefactor as calculated in DFPT [23] and avoid its fitting on the experimental data. The objective of this KMC will also be to account for the disparity of all activation barriers calculated above. It will then be necessary to determine lifetimes for each of these configurations, to construct energy basins, for simpler, more efficient but accurate implementation in commercial TCAD tools, such as using and refining a single activation barrier for both migration and dissociation mechanisms. 


\section{ACKNOWLEDGEMENTS}

This work was performed using HPC resources from CALMIP-Grant P1555. The research leading to these results has received funding from the European Union's Horizon 2020 research and innovation program under grant agreement No. 871813 MUNDFAB. A. Jay, M. Gunde, N. Salles, L. MartinSamos and A. Hémeryck are active members of the Multiscale and Multi-Model Approach for Materials in Applied Science consortium (MAMMASMIAS consortium), and acknowledge the efforts of the consortium in fostering scientific collaboration.

\section{REFERENCES}

[1] S. Glunz, S. Rein, W. Warta, J. Knobloch, and W. Wettling, "On the degradation of cz-silicon solar cells," in Proc. 2nd WC PVSEC, 1998.

[2] T. Mchedlidze and J. Weber, "Direct detection of carrier traps in si solar cells after light-induced degradation," physica status solidi (RRL)-Rapid Research Letters, vol. 9, no. 2, pp. 108-110, 2015.

[3] X. Yu, P. Wang, P. Chen, X. Li, and D. Yang, "Suppression of boronoxygen defects in p-type czochralski silicon by germanium doping," Appl. Phys. Lett., vol. 97, no. 5, p. 051903, 2010.

[4] "Sentaurus process user guide, 2019.03, synopsis inc."

[5] M. Vaqueiro-Contreras, V. P. Markevich, J. Coutinho, P. Santos, I. F. Crowe, M. P. Halsall, I. Hawkins, S. B. Lastovskii, L. I. Murin, and A. R. Peaker, "Identification of the mechanism responsible for the boron oxygen light induced degradation in silicon photovoltaic cells," J. App. Phys., vol. 125, no. 18, p. 185704, 2019.

[6] X. Chen, X. Yu, X. Zhu, P. Chen, and D. Yang, "First-principles study of interstitial boron and oxygen dimer complex in silicon," Applied Physics Express, vol. 6, no. 4, p. 041301, 2013.

[7] P. Hohenberg and W. Kohn, "Inhomogeneous electron gas," Phys. Rev., vol. 136, p. B864, 1964.

[8] W. Kohn and S. L. J, "Self-consistent equations including exchange and correlation effects," Phys. Rev., vol. 140, p. A1133, 1965.

[9] J. P. Perdew, K. Burke, and M. Ernzerhof, "Generalized gradient approximation made simple," Phys. Rev. Let., vol. 77, no. 18, p. 3865, 1996.

[10] P. Giannozzi, S. Baroni, N. Bonini, M. Calandra, R. Car, C. Cavazzoni, D. Ceresoli, G. L. Chiarotti, M. Cococcioni, I. Dabo, A. Dal Corso, S. de Gironcoli, S. Fabris, G. Fratesi, R. Gebauer, U. Gerstmann, C. Gougoussis, A. Kokalj, M. Lazzeri, L. Martin-Samos, N. Marzari, F. Mauri, R. Mazzarello, S. Paolini, A. Pasquarello, L. Paulatto, C. Sbraccia, S. Scandolo, G. Sclauzero, A. P. Seitsonen, A. Smogunov, P. Umari, and R. M. Wentzcovitch, "Quantum espresso: a modular and open-source software project for quantum simulations of materials," $J$. Phys.: Cond. Matter, vol. 21, p. 395502, 2009.

[11] J. Heyd, G. E. Scuseria, and M. Ernzerhof, "Hybrid functionals based on a screened coulomb potential," J. Chem. Phys., vol. 118, no. 18, pp. 8207-8215, 2003.

[12] H. Monkhorst and J. Pack, "Special points for Brillouin-zone integrations," Phys. Rev. B, vol. 13, p. 5188, 1976.

[13] A. Jay, C. Huet, N. Salles, M. Gunde, L. Martin-Samos, N. Richard, G. Landa, V. Goiffon, S. De Gironcoli, A. Hémeryck, and N. Mousseau, "Finding reaction pathways and transition states: r-artn and d-artn as an efficient and versatile alternative to string approaches," J. Chem. Theory Comput., vol. 16, pp. 6726-6734, 2020.

[14] F. Livingston, S. Messoloras, R. Newman, B. Pike, R. Stewart, M. Binns, W. Brown, and J. Wilkes, "An infrared and neutron scattering analysis of the precipitation of oxygen in dislocation-free silicon," Journal of Physics C: Solid State Physics, vol. 17, no. 34, p. 6253, 1984.

[15] Z. Zeng, J. Murphy, R. Falster, X. Ma, D. Yang, and P. Wilshaw, "The effect of impurity-induced lattice strain and fermi level position on low temperature oxygen diffusion in silicon," J. App. Phys., vol. 109, no. 6, p. 063532, 2011.

[16] J. Coutinho, R. Jones, P. Briddon, and S. Öberg, "Oxygen and dioxygen centers in si and ge: Density-functional calculations," Phys. Rev. B, vol. 62, no. 16, p. 10824, 2000.

[17] R. Newman, "Oxygen diffusion and precipitation in czochralski silicon," J. Phys.: Cond. Matter, vol. 12, no. 25, p. R335, 2000.
[18] M.-H. Du, H. M. Branz, R. S. Crandall, and S. Zhang, "Bistabilitymediated carrier recombination at light-induced boron-oxygen complexes in silicon," Phys. Rev. Let., vol. 97, no. 25, p. 256602, 2006.

[19] L. Murin, T. Hallberg, V. Markevich, and J. Lindström, "Experimental evidence of the oxygen dimer in silicon," Phys. Rev. Let., vol. 80, no. 1, p. 93, 1998.

[20] D. Tsoukalas and P. Ciienevier, "Boron diffusion in silicon by a vacancy mechanism," Phys. Sol. Stat., vol. 92, pp. 495-501, 1985.

[21] I. Martin-Bragado, P. Castrillo, M. Jaraiz, R. Pinacho, J. Rubio, and J. Barbolla, "Physical atomistic kinetic monte carlo modeling of fermilevel effects of species diffusing in silicon," Phys. Rev. B, vol. 72, no. 3, p. 035202, 2005.

[22] M. Gunde, N. Salles, N. Richard, A. Hemeryck, and L. Martin Samos, "An off-lattice kinetic monte carlo kernel guided by topological and geometrical analysis to bridge accurate ab-initio calculations and large scale simulations," Bulletin of the American Physical Society, 2021.

[23] S. Baroni, S. de Gironcoli, A. Dal Corso, and P. Giannozzi, "Phonons and related crystal properties from density-functional perturbation theory," Rev. Mod. Phys., vol. 73, p. 515, 2001. 